\title{
Urteil vom 29. Mai 2009: kein Leitentscheid für die Einführung von SwissDRG
}

\section{Hanspeter Kuhn}

Fürsprecher,

stv. Generalsekretär der FMH

* Die ausführlichen Belege in den Fussnoten sind in der Online-Version unter www.saez.ch $\rightarrow$ Aktuelle Nummer oder Archiv $\rightarrow$ $2011 \rightarrow 16 / 17$ nachzulesen.

Korrespondenz: Rechtsdienst FMH Postfach 170 CH-3000 Bern 15 Tel. 0313591111 Fax 0313591112 lex@fmh.ch
Man hört oft, das Urteil des Bundesverwaltungsgerichts vom 29. Mai 2009 [1]* zum Berner Spitaltarifvertrag sei ein Leitentscheid für die Umsetzung der Revision Spitalfinanzierung und damit für die Einführung von SwissDRG. Auch die Einladung des BAG zum Hearing vom 28.3.2011 enthielt einen entsprechenden Hinweis [2].

Dem liegt ein Missverständnis zugrunde. Das Urteil betraf einen Tarifvertrag vom 15. September 2006 [3]. Dieser Tarifvertrag war somit abgeschlossen worden, noch bevor das Parlament die KVG-Revision zur Spitalfinanzierung zu Ende beraten hatte. Das Gericht hat deshalb die Vertragsinhalte ausdrücklich und zu Recht nach dem alten KVG von 1994 beurteilt, und nicht gemäss der KVG-Revision Spitalfinanzierung [4]. Es hat richtigerweise auch die alte Fassung von Art. $59 \mathrm{KVV}$ herangezogen [5].

Für die Umsetzung der KVG-Revision Spitalfinanzierung und die Einführung von SwissDRG sind hingegen die parlamentarischen KVG-Beratungen der letzten Jahre zu berücksichtigen. Und die sind interessant.

- In der KVG-Revision Spitalfinanzierung wurde 2007 tatsächlich der Antrag gestellt, der genau das beinhaltete, was die Krankenversicherer heute fordern: «Bei leistungsbezogenen Fallpauschalen muss der Leistungserbringer auf der Rechnung alle Angaben machen, welche für die Ermittlung der korrekten Fallpauschalen notwendig sind, insbesondere auch die relevanten Diagnosen und Prozeduren. Der Bundesrat erlässt die Bestimmungen zur Sicherstellung des Datenschutzes.» Pikant: Der Nationalrat hat diesen Antrag im September 2007 mit 94 zu 79 abgelehnt [6]. Der heute geltende Gesetzestext zur Spitalfinanzierung verlangt denn auch nicht, dass auf der Rechnung die relevanten Diagnosen und Prozeduren stehen müssten und dürften.

- Im Rahmen der Beratung der dringlichen Massnahmen gegen die Kostensteigerung gelang es dann den Vertretern der Krankenversicherer zwar, über den Ständerat einen neuen Art. 42 Abs. 3bis KVG einzubringen mit dem Wortlaut: «Die Leistungserbringer haben auf der Rechnung nach Absatz 3 die Diagnosen nach der Internationalen Klassifikation der Krankheiten in der jeweiligen vom zuständigen Departement herausgegebenen schweizerischen Fassung codiert aufzuführen.
Der Bundesrat erlässt nähere Vorschriften zur Erhebung, Bearbeitung und Weitergabe der Daten unter Wahrung des Verhältnismässigkeitsprinzips.» [7].

Doch hat das Parlament diese Gesetzesvorlage in der Schlussabstimmung abgelehnt.

Es ist in der Schweiz üblich, die Gesetze anzuwenden, die das Parlament beschlossen hat. Nicht üblich ist es hingegen, abgelehnte Anträge oder in der Schlussabstimmung gescheiterte Vorlagen als geltendes Recht anzuwenden. Aufgrund der Gesetzgebungsgeschichte der letzten Jahre ist klar: Das heutige KVG erlaubt keine systematische Weitergabe von detaillierten Diagnosen und Prozeduren, auch und insbesondere nicht im Kontext von SwissDRG [8].

\section{Literatur}

1 C-6570/2007.

2 Einladung vom 28.2.2011 zu einer Aussprache betreffend Datenübermittlung: «(...) Massgebend dafür ist das Grundsatzurteil des Bundesverwaltungsgerichts vom 29. Mai 2009 in Sachen santésuisse gegen den Regierungsrat des Kantons Bern (C-6570/2007).»

3 A. «Santésuisse (...) hat am 15. September 2006 mit A (...) einen Tarifvertrag (...) abgeschlossen.»

4 E. 2.1 .

5 E. 3.3 .4 .2

6 Ablehnung Antrag Stahl nach folgenden Voten u.a. von Bundesrat Couchepin: «Le problème est de savoir quelles données doivent systématiquement apparaître sur la facture dans le sens d'un rminimal data set. (...) ... nous pensons aussi que, dans le doute, il faut trancher en faveur de la protection des données plutôt qu'en faveur du confort des assureurs-maladie...» (AB 2007 N 445); Kommissionssprecher Guisan: «Avec les DRG et l'étalonnage (benchmarking), les assureurs vont bénéficier d'instruments extrêmement performants pour déterminer les situations potentiellement abusives.» (AB 2007 N 446)

7 Vergessen wurden in diesem Antrag hingegen die von den Krankenkassen heute auch geforderten Prozeduren.

8 Auch der heutige Wortlaut von Art. 59 KVV und der Kommentar (vom 22.10.2008, siehe Seite $9 \mathrm{zu}$ Rechnungsstellung Artikel 59) sehen - richtigerweise nicht vor, dass bei DRG die detaillierten Diagnosen und Prozeduren auf der Rechnung stehen müssten. 


\section{Urteil vom 29. Mai 2009: kein Leitentscheid für die Einführung von SwissDRG}

\section{Hanspeter Kuhn, Fürsprecher, stv. Generalsekretär}

Man hört oft, das Urteil des Bundesverwaltungsgerichts vom 29. Mai $2009^{1}$ zum Berner Spitaltarifvertrag sei ein Leitentscheid für die Umsetzung der Revision Spitalfinanzierung und damit für die Einführung von SwissDRG. Auch die Einladung des BAG zum Hearing vom 28.3.2011 enthielt einen entsprechenden Hinweis. ${ }^{2}$

Dem liegt ein Missverständnis zugrunde. Das Urteil betraf einen Tarifvertrag vom 15. September $2006 .{ }^{3}$ Dieser Tarifvertrag war somit abgeschlossen worden, noch bevor das Parlament die KVG-Revision zur Spitalfinanzierung zu Ende beraten hatte. Das Gericht hat deshalb die Vertragsinhalte ausdrücklich und zu Recht nach dem alten KVG von 1994 beurteilt, und nicht gemäss der KVG-Revision Spitalfinanzierung: ${ }^{4}$ Es hat richtigerweise auch die alte Fassung von Art 59 KVV herangezogen. ${ }^{5}$

Für die Umsetzung der KVG-Revision Spitalfinanzierung und damit für die Einführung von SwissDRG sind hingegen die parlamentarischen KVG-Beratungen der letzten Jahre zu berücksichtigen. Und die sind interessant.

a) In der KVG-Revision Spitalfinanzierung wurde 2007 tatsächlich der Antrag gestellt, der genau das beinhaltete, was die Krankenversicherer heute fordern: «Bei leistungsbezogenen Fallpauschalen muss der Leistungserbringer auf der Rechnung alle Angaben machen, welche für die Ermittlung der korrekten Fallpauschalen notwendig sind, insbesondere auch die relevanten Diagnosen und Prozeduren. Der Bundesrat erlässt die Bestimmungen zur Sicherstellung des Datenschutzes »

Pikant: Der Nationalrat hat diesen Antrag im September 2007 mit 94 zu 79 abgelehnt. ${ }^{6}$ Der heute geltende Gesetzestext zur Spitalfinanzierung verlangt denn auch nicht, dass

${ }_{2}^{1}$ C-6570/2007.

2 Aus der Einladung vom 28.2.2011 zu einer Aussprache betreffend Datenübermittlung für das Hearing vom 28.3.2011: „Die Tarifpartner wurden namentlich aufgefordert, die Übermittlung von Diagnose- und Prozedurencodes respektive die Rechnungstellung vor der Einführung der leistungsbezogenen Pauschalen konkret zu regeln. Massgebend dafür ist das Grundsatzurteil des Bundesverwaltungsgerichts vom 29. Mai 2009 in Sachen santesuisse gegen den Regierungsrat des Kantons Bern (C-6570/2007).“

${ }^{3}$ A. ,Santésuisse, Die Schweizer Krankenversicherer, handelnd durch santésuisse, Geschäftsstelle Bern (nachfolgend: santésuisse) hat am 15. September 2006 mit A.____ (beziehungsweise mit B.____ als deren Rechtsvorgängerin) einen Tarifvertrag gemäss Art. 46 des Bundesgesetzes vom 18. März 1994 über die Krankenversicherung (KVG, SR 832.10) abgeschlossen betreffend die Behandlung von stationären Akutpatienten der allgemeinen Abteilung in öffentlichen Spitälern des Kantons Bern.“

${ }^{4}$ „E. 2.1 Die obligatorische Krankenpflegeversicherung übernimmt nach Art. 24 KVG (nachfolgend wird das KVG in der bis zum 31. Dezember 2008 geltenden Fassung [also gerade nicht die Vorlage Spitalfinanzierung; Anmerkung des Autors] zitiert, da betreffend das materielle Recht auf den Zeitpunkt der Verfügung abzustellen ist [ULRICH HÄFELIN/GEORG MÜLLER/FELIX UHLMANN, Allgemeines Verwaltungsrecht, 5. Aufl., Zürich u.a. 2006, Rz. 326 f.]) die Kosten für die Leistungen gemäss Art. 25-31 KVG nach Massgabe der in den Art. 32-34 KVG festgelegten Voraussetzungen.“

5 „E. 3.3.4.2 In Art. 59 KVV regelt der Bundesrat, entsprechend dem Auftrag von Art. 42 Abs. 3 KVG, die Einzelheiten der Rechnungstellung. Nach Art. 59 Abs. 1 KVV haben die Leistungserbringer in ihren Rechnungen folgende Angaben zu machen: a) Kalendarium der Behandlungen; b) erbrachte Leistungen im Detaillierungsgrad, den der massgebliche Tarif vorsieht; c) Diagnosen im Rahmen von Abs. 2; dieser bestimmt seinerseits, dass tarifvertraglich vereinbart werden kann, welche Angaben und Diagnosen in der Regel nur dem Vertrauensarzt bekannt zu geben sind, und dass sich die Weitergabe der Diagnose ferner nach Art. 42 Abs. 4 und 5 KVG richtet." [Auch hier: Dies ist die KVV-Fassung vor den Ergänzungen für die Umsetzung der KVG-Revison Spitalfinanzierung]

${ }^{6}$ Der Antrag Stahl (AB 2007 N 442) nahm den - in der Folge zurückgezogenen - Antrag Humbel Näf auf und ergänzte diesen mit dem Auftrag an den Bundesrat, den Datenschutz zu regeln: Die Debatte im Nationalrat erkannte klar die Bedeutung des Antrags, siehe u.a. die Voten von BR Couchepin und Kommissionssprecher Guisan: 
auf der Rechnung die relevanten Diagnosen und Prozeduren stehen müssten und dürften.

b) Im Rahmen der Beratung der dringlichen Massnahmen gegen die Kostensteigerung gelang es dann den Vertretern der Krankenversicherer zwar, über den Ständerat einen neuen Art. 42 Abs. 3bis KVG einzubringen mit dem Wortlaut: „Die Leistungserbringer haben auf der Rechnung nach Absatz 3 die Diagnosen nach der Internationalen Klassifikation der Krankheiten in der jeweiligen vom zuständigen Departement herausgegebenen schweizerischen Fassung codiert aufzuführen. Der Bundesrat erlässt nähere Vorschriften zur Erhebung, Bearbeitung und Weitergabe der Daten unter Wahrung des Verhältnismässigkeitsprinzips.“7

Doch hat das Parlament diese Gesetzesvorlage in der Schlussabstimmung abgelehnt.

Es ist in der Schweiz üblich, die Gesetze anzuwenden, die das Parlament beschlossen hat. Nicht üblich ist es hingegen, abgelehnte Anträge oder in der Schlussabstimmung gescheiterte Vorlagen als geltendes Recht anzuwenden. Aufgrund der Gesetzgebungsgeschichte der letzten Jahre ist klar: Das heutige KVG erlaubt keine systematische Weitergabe von detaillierten Diagnosen und Prozeduren, auch und insbesondere nicht im Kontext von SwissDRG. ${ }^{8}$

Bundesrat Couchepin : « [...] Les systèmes de la tarification à la prestation, tels que le système DRG, se basent par définition sur le diagnostic et attribuent les cas à des groupes plus ou moins homogènes. En fonction de ces attributions, le diagnostic peut être posé, au moins en ce qui concerne le groupe, même si ce n'est pas toujours le cas pour chaque individu. Dans ce cas, la décision en faveur du système de tarification lié à la prestation implique que certaines indications concernant le diagnostic apparaîtront sur la facture pour permettre leur contrôle, comme l'exige l'article 42 de la LAMal. Le problème est de savoir quelles données doivent systématiquement apparaître sur la facture dans le sens d'un "minimal data set".

Madame Humbel Näf voudrait que toutes les indications apparaissent sur toutes les factures, sous réserve de ce qui relève de la protection des données avec le complément de la proposition Stahl à l'alinéa 4bis. Nous craignons que cela n'aboutisse à une telle abondance d'informations que cela ne soit finalement plus proportionnel aux besoins des assureurs.

Nous sommes convaincus que la solution du Conseil fédéral et de la majorité est suffisante pour permettre aux assureurs de faire leur travail, même sans la solution Stahl, et nous pensons aussi que, dans le doute, il faut trancher en faveur de la protection des données plutôt qu'en faveur du confort des assureurs-maladie, confort relatif puisqu'ils recevraient tellement d'informations qu'ils ne seraient plus en état de les maîtriser. C'est une question de nuances: nous sommes dans un état libéral et tranchons plutôt en faveur de la protection des données qu'en faveur des assureurs. » (AB $2007 \mathrm{~N} 444 f)$

Yves Guisan als Kommissionssprecher : «[...] Exiger que tous les éléments déterminants des DRG figurent sur la facture va manifestement à l'encontre de la protection des données, cela a déjà été dit, d'autant plus qu'ils seront traités par un collaborateur et pas nécessairement par le médecin-conseil.

Cette situation est déjà hautement problématique en soi. De plus, la gestion de cette masse impressionnante de données va obliger à un développement considérable de l'infrastructure informatique et administrative, donc engendrer aussi des coûts supplémentaires absolument considérables. Cela correspond à une conception manifestement dépassée des contrôles. Avec les DRG et l'étalonnage (benchmarking), les assureurs vont bénéficier d'instruments extrêmement performants pour déterminer les situations potentiellement abusives. D'autre part, les médecins-conseils ont toujours la possibilité de demander des renseignements complets face à un cas particulier qui pourrait faire problème - Monsieur le conseiller fédéral Couchepin vient de le rappeler.

Si l'on accepte la proposition de la minorité Humbel Näf, ou même la proposition Stahl, c'est la fin du système du médecin-conseil. » (AB 2007 N 445)

Der Antrag Stahl wurde mit 94 zu 79 abgelehnt . (AB 2007 N 446).

${ }^{7}$ Vergessen wurden in diesem Antrag die von den Krankenkassen heute zudem geforderten Prozeduren.

${ }^{8}$ Auch der Wortlaut von Art. $59 \mathrm{KVV}$ sieht - richtigerweise - nicht vor, dass bei DRG die detaillierten Diagnosen und Prozeduren auf der Rechnung stehen müssten. Die DRG-bezogenen Ergänzungen der KVV lauten seit 1.1.2009 wie folgt:

„1bis Für die Bearbeitung der diagnosebezogenen Daten treffen die Versicherer die nach Artikel 20 der Verordnung vom 14. Juni 1993 zum Bundesgesetz über den Datenschutz erforderlichen technischen und organisatorischen datensichernden Massnahmen.

1ter Zur Aufbewahrung der diagnosebezogenen Daten werden die Personalien der Versicherten pseudonymisiert. Die Aufhebung der Pseudonymisierung kann nur durch den Vertrauensarzt oder die Vertrauensärztin des Versicherers erfolgen." 
Auch der Kommentar des Bundesrats hält nirgendwo fest, es müssten systematisch detaillierte Diagnosen und Prozeduren auf der Rechnung stehen. Die entscheidende Passage lautet:

„Die diagnosebezogenen Angaben sind besonders schützenswerte Personendaten. Die Versicherer müssen dafür besorgt sein, dass deren Schutz gewährleistet ist. Die diesbezüglichen technischen und organisatorischen Anforderungen sind in den Artikeln 8-10 und 20-22 der Verordnung vom 14. Juni 1993 zum Bundesgesetz über den Datenschutz (SR 235.11) verankert. Weil die Aufbewahrung der Daten nicht geregelt ist, wird in Absatz 1 ter festgehalten dass die Daten in pseudonymisierter Form aufzubewahren sind und dass die Pseudonymisierung nur durch den Vertrauensarzt des Versicherers aufgehoben werden kann." (nicht datierter Kommentar zur Änderung der KVV vom 22.10.2008, Seite 9 zu Rechnungsstellung Artikel 59.) 